\title{
La identidad (habitus) y la intersubjetividad de los comerciantes de los mercados móviles de la Ciudad de México como coadyuvantes en la participación de sus asambleas y construcción del capital social
}

\author{
The identity (habitus) and the intersubjectivity of the merchants of \\ the street markets of Mexico City as adjuvants in the participation \\ of their assemblies and building of the social capital
}

Guillermo García Mayo*

Investigador independiente

Guanajuato núm. 100, Col. Roma Norte,

C.P. 06700, Ciudad de México, México

Editor: Rogelio del Prado Flores

Fecha de recepción: 6 de septiembre de 2021

Fecha de aceptación: 2 de diciembre de 2021 guiller67_@hotmail.com

https://orcid.org/0000-0003-1509-2588

\section{RESUMEN}

La presente investigación se propone averiguar cómo la identidad (babitus) y la intersubjetividad de los comerciantes de los mercados móviles coadyuvan a la participación en las asambleas y a crear un capital social sólido que beneficie a toda la comunidad. En este sentido, existen problemas entre comerciantes que afectan la convivencia y funcionamiento de los bazares; el género como parte de la identidad de los comerciantes puede contribuir al mejoramiento. La metodología que se empleó fue mixta, se aplicaron 152 encuestas a comerciantes del Bazar del Oro y a II 5 comerciantes del Bazarte Obregón, asimismo, se entrevistaron a cinco comerciantes de cada uno de los bazares. Los resultados obtenidos muestran que los comerciantes presentan una contradictoria identidad (babitus) e intersubjetividad, siendo en un bazar fundamento aceptable para participar en las asambleas y mejorar el capital social, pero para otro es todo lo contrario.

Palabras clave: identidad, habitus, intersubjetividad, capital social, comerciantes, asambleas.

* Licenciado en Mercadotecnia y Maestro en Comercio Exterior por la Universidad del Valle de México, Doctor en Administración Pública por la Universidad Anáhuac México, Campus Norte. Se ha desempañado laboralmente en la Secretaría de la Defensa Nacional, en la Procuraduría General de la República y como Director General de Comercialización en la empresa de análisis clínicos Unidad de Diagnóstico Microbiológico (UDMSC). 


\begin{abstract}
This research aims to find out how the identity (babitus) and intersubjectivity of merchants in street markets contribute to participation in assemblies and create a solid social capital that benefits the entire community. In this sense, there are problems between merchants that affect the coexistence and operation of the bazaars; gender as part of the identity of merchants can contribute to improvement. The methodology used was mixed, I52 surveys were applied to merchants from the Bazar del Oro and II5 merchants from Bazarte Obregón, likewise, five merchants from each of the bazaars were interviewed. The results obtained show that merchants present a contradictory identity (babitus) and intersubjectivity, being in a bazaar an acceptable foundation to participate in assemblies and improve social capital, but for another it is the opposite.
\end{abstract}

Keywords: identity, habitus, intersubjectivity, social capital, merchants, assemblies.

\title{
INTRODUCCIÓN
}

La presente investigación se desarrolló con una metodología mixta comparativa de dos mercados móviles de la Ciudad de México, siendo el Bazar del Oro y el Bazarte Obregón, ubicados en las colonias Roma Norte y Roma, Sur respectivamente, de la Alcaldía Cuauhtémoc. El objetivo es saber si la identidad (babitus) e intersubjetividad de los comerciantes contribuyen en la participación de las asambleas que realizan las asociaciones civiles y en la cimentación de un capital social sólido que ayude al bienestar de sus integrantes. La importancia de investigar estos dos bazares es porque son emblemáticos de la zona y lugares asiduos para comprar y convivir por familias los fines de semana. Se consultaron fuentes científicas especializadas en trabajos de investigación sobre mercados móviles; en esta búsqueda se cubrieron las variables sobre la identidad (babitus), intersubjetividad y capital social, así como las peculiaridades de los comerciantes de los mercados móviles como el género.

De la misma manera, se antepuso pleno interés en los objetivos establecidos, metodologías empleadas, referentes teóricos sustentados, resultados encontrados y conclusiones puntualizadas; qué establecieron, encontraron y dedujeron los autores de las diferentes investigaciones examinadas. También se revisó la teoría existente contemporánea y actualizada sobre investigadores y autores expertos en identidad (babitus), comunicación y especializados en intersubjetividad en diversos contextos situacionales. De la misma manera, se revisó la teoría existente sobre capital social y cómo se han modificado los diversos conceptos, que para la presente investigación ayudan a tener un marco teórico y contextual más formal. 
Al momento de aplicar las encuestas y realizar las entrevistas a los comerciantes de ambos bazares, los hallazgos proporcionaron datos interesantes, particulares y característicos de cada bazar, que al ser comparados se desprende información preliminar trascendental ofreciendo un panorama exclusivo y contrapuesto en cada uno de los mercados móviles. En el análisis y discusión se observa una correlación inversamente proporcional en los resultados de las investigaciones cuantitativas y cualitativas, dejando en claro que los comerciantes de cada bazar cuentan con capital social caracterizado por la identidad (babitus) e intersubjetividad.

\section{INVESTIGACIONES CIENTÍFICAS SOBRE IDENTIDAD}

(HABITUS) Y CAPITAL SOCIAL

En el presente apartado se busca saber cómo la identidad (babitus) y el capital social de los comerciantes en los mercados móviles se produce por las condiciones sociodemográficas, historias de vida y cultura; en este sentido, se aclara que la identidad y babitus no son semejantes; el babitus interviene en la identidad de las personas redefiniendo la interacción entre ellas, es el fundamento objetivo del estado subjetivo de la identidad. Para Garza (2019), la delimitación de sus recursos culturales pueden producir cambios en el entorno social, ya sea que se refuerce la identidad colectiva, que a consideración de Esquivel (2019) busca demostrar que la identidad puede transformar la percepción social del espacio donde se da, repercutiendo en una mejoría de la identidad social; en este contexto, la sociedad se rehace permanentemente para González (20I9) porque se integran visiones de distintos actores en una unidad social y se identifican las diferencias de identidades, que en opinión de Sánchez (2019) examina la forma en que las prácticas promueven actitudes de innovación, en cambio, con una visión diferente Feregrino y Cadena (2019) analizan la identidad de personas que trabajan en el espacio público con la perspectiva de género, experiencias y significados que se construyen en el proceso de envejecimiento y en el desarrollo laboral y social del ser humano; que de otra manera, López (2018) pretende comprobar que las identidades de los comerciantes de un mercado, son la representación de sus relaciones sociales ante las medidas que impone el Estado.

Esquivel (2019), Garza (2019) y Sánchez (2019), coinciden en sus indagaciones en el método mixto de investigación para recabar datos cualitativos y cuantitativos, por la parte cualitativa se le da una relevancia a las entrevistas en profundidad con preguntas semiestructuradas, así como observaciones participantes y pláticas informales; por la parte cuantitativa se emplean encuestas, muestreo situacional y análisis estadístico; asimismo, existe una tendencia hacia la etnografía, que a juicio de López (20I8), convergen en este método 
para investigar la forma de vida de una unidad social por medio de la interpretación y comprensión de los actores, sociedades y culturas; sin embargo, González (2019) y Feregrino y Cadena (2019) prefieren únicamente la metodología cualitativa, aplicando entrevistas en profundidad para estudiar la percepción diagnóstica desde cada enfoque que tienen los diferentes grupos de entrevistados y de sus narrativas construidas, abordando la experiencia como vivencia y realidad.

Los referentes teóricos en que se fundamentan Esquivel (2019), Sánchez (2019) y González (2019), distinguen al capital social como elemento central de las redes sociales, produciendo la identidad cuando un individuo se identifica con un grupo de personas de culturas afines, además, muestra que el capital social es una parte importante de la estructura social, que en opinión de Sánchez (2019), implanta que el capital social facilita prácticas que forman parte de una misma cultura y determina que los fenómenos sociales son explicados desde el enfoque social, en cambio, González (2019) averigua que los hechos sociales son las maneras de obrar, pensar y sentir del individuo, por tanto, pueden explicarse a partir de la cultura, observando manifestaciones individuales y colectivas que caracterizan el desarrollo de una sociedad en un contexto, asimismo, para Garza (2019) lo sociocultural es como un pilar del desarrollo, donde se desarrolla el concepto de sostenibilidad cultural, integrando valores como la cohesión social, la identidad, el sentido de pertenencia y la resiliencia, que a juicio de López (2018), la identidad permite asumir a un sujeto su propia conciencia, conceptualizando que en un determinado momento y contexto, una persona tiene conciencia, y esta se muestra en la capacidad de diferenciarse y parecerse social y culturalmente de otros, en cambio, Feregrino y Cadena (2019), presentan las transiciones heterogéneas de intensidad y duración, como cambios subjetivos en un momento específico, introduciendo que las rutas de vida de las personas, al ser influenciadas por componentes sociales, no ocurren con una tendencia lineal.

López (2018) halla que los comerciantes le dan sentido de existencia al comercio debido a sus prácticas sociales internas, relacionándose diversas identidades para dar por entendido prácticas comerciales peculiares, que a consideración de los resultados de Esquivel (2019) y Sánchez (2019), las identidades ayudan a la transformación de los espacios sociales y de las relaciones sociales de las personas, las que se organizan en un sistema productor de identidades y realidades; en cambio Garza (2019) y González (2019) encuentran que dentro del espacio social existen redes sociales con identidades específicas muy heterogéneas que no pueden ser valorizados con un enfoque generalizado, en este mismo contexto, el proceso de construcción de la identidad para Feregrino y Cadena (2019) es constante y dinámico, descubriendo que toda persona tiene identidad y quiere pertenecer a grupos sociales semejantes a sus características afines de identidad. 
Las investigaciones de Garza (2019), Esquivel (2019), González (2019) y Sánchez (2019), buscan mejorar el espacio social comercial por medio de las relaciones sociales e identidades de los actores, que pueden ser semejantes o distintas; de la misma manera, Esquivel (2019), Garza (2019) y Sánchez (2019), emplean la metodología mixta, recabando datos cuantitativos y cualitativos, en este último método, Feregrino y Cadena (2019) emplean las entrevistas en profundidad en específico la técnica de historia de vida, mientras López (20I8) emplea la etnografía para estudiar la forma como vive una sociedad en determinado contexto. Todos los resultados de los artículos estudiados coinciden en que las identidades y prácticas del capital social de los participantes proporcionan al mercado una existencia personalizada.

\section{LA INTERSUBJETIVIDAD DE LOS COMERCIANTES}

\section{EN LOS MERCADOS MÓVILES}

Los mercados móviles son “(...) un canal de distribución y abasto popular de productos pertenecientes principalmente a la canasta básica, el cual se instala un día o más en la vía pública" (Gaceta Oficial de la Ciudad de México [GOCDMX], 20I9, p.5), se encuentran inmersos en espacios urbanos complejos de multiculturalidad, donde lo que se desea es afectar lo menos posible a la sociedad y ofrecer un espacio agradable y cordial al ambiente donde se establecen (Jackson, 2019). Abordar el estudio de la intersubjetividad en los mercados móviles representa estudiar categorías de la comunicación, en específico la comunicación interpersonal que se da a partir de dos personas hasta un número limitado no establecido en forma estricta, donde la integración e interacción entre ellos trata de construir entendimientos compartidos y significaciones mutuas de relaciones (Habermas, 20I4). Es así como toda acción humana social de manera inevitable se posibilita por medio del lenguaje que, a su vez, es una acción social, intersubjetiva (Schütz, 1972), en el que una persona que tiene conciencia se comunica con otra u otras personas.

El reconocimiento mutuo entre los comerciantes y la comprensión de que existe otra conciencia es el fundamento de la creación del mundo intersubjetivo, pero esto no se presenta de forma natural, es decir, en el espacio que se da o existe entre los comerciantes que en un momento dado se reconocen, se produce el proceso de construcción cooperativa de los significados intersubjetivos del lenguaje en general, de tal manera que el reconocimiento de la otra persona como sí mismo y de sí mismo como otra persona es un cumplimiento de la intersubjetividad y la comunicación, organizando a las personas como personas morales o físicas (De Zan, 20I7). Entonces se asume que la intersubjetividad permite en cualquier 
relación social la posibilidad de una construcción de relación equilibrada con capacidad de reflexionar, pensar y deducir con otras personas (Dussel, 200I), por tanto, lo intersubjetivo se construye de manera colectiva, buscando cimentar sentidos, plantear significados y alcanzar acuerdos con base en las interacciones con significados intersubjetivos por medio de la comunicación en un contexto establecido (Schütz, I972) que posibiliten la construcción de fuentes de saber que integran la cultura, sociedad y personalidad de los individuos, que a su vez, el mundo de la vida se instala en ellos y se recogen y conforman ilaciones hermenéuticas para comprender las circunstancias, permitiendo tomar acuerdos en beneficio de las suposiciones, ideas y percepciones comunes para la conformación de la identidad (Uribe et al., 2020).

De lo anterior el mundo de la vida cuenta con componentes estructurales, los cuales se conciben como espacios que se relacionan estableciendo "pautas interpretativas o suposiciones sobre cómo la cultura ejerce una influencia sobre la acción, sobre cómo se van estableciendo pautas determinadas en las interacciones sociales de los sujetos y finalmente, cómo se estructura un modo de ser de los individuos" (Garrido, 2oII, p. 6), donde se intercambia información de manera interactiva, intencional y multidimensional en el contexto de una relación y con consecuencias en las relaciones (Hernández-Jorge y De La Rosa, 20I7), respondiendo a las necesidades de las personas por comunicarse de acuerdo con factores ambientales y precisando de la retroalimentación verbal y no verbal, intencionada y no intencionada, multifuncional, transaccionaly reveladora (García M., 2015), manifestando que es una comunicación cara a cara.

Evidentemente la intersubjetividad y la comunicación interpersonal son diferentes. Entre los comerciantes se hace interpersonal cuando ellos se encuentran y se sienten en un ambiente o contexto que se presta para compartir su humanidad (Stewart, Zediker, y Witteborn, 2004), siendo verbal y no verbal la interacción (DeVito, 20I3) e inspirando una conexión (Solomon y Theiss, 2013). En cambio, durante los encuentros diarios entre los comerciantes, donde se reconocen y están conscientes de la otredad, construyen el mundo desde un enfoque particular, estableciendo significados compartidos con la comunidad en un mundo organizado, es decir, la persona en el encuentro con otras conciencias en un espacio construyen su intersubjetividad, la visión del mundo desde su exclusiva perspectiva en común consiguiendo la construcción de una realidad social en particular, donde los hábitos personales se convierten en hábitos públicos, siendo aceptados y entendidos por medio dela comunicación interpersonal que pone en común (Garza, 2009) las interacciones cotidianas de convivencia. 


\section{LA IDENTIDAD (HABITUS) DE LOS COMERCIANTES}

\section{Y EL CAPITAL SOCIAL}

Los valores de los comerciantes son cambiantes y el hábito del latín babitus, como costumbre, significan modos, maneras, estilos, rutinas, etc., que caracterizan a una persona. Pierre Bourdieu lo define como el "sistema de disposiciones adquiridas por medio del aprendizaje implícito o explícito que funciona como un sistema de esquemas" (I990, p. I57). Entonces la identidad (babitus) obedece a las reglas del campo o espacio donde se esté jugando; este espacio, fija los límites por la disputa de una identidad (Foucault, 2008). La identidad principalmente se constituye en lo infinito de la presencia del otro, quien es un ser humano separado y diferente de la identidad del yo. Una y otra identidad tienen una separación, aunque tengan una presencia en común en un espacio abstracto y temporalidad imprecisa, donde la unión superflua, invitan más a la separación sin ninguna justificación en contrario. Esta relación con la otra identidad no demuestra o compromete el babitus del yo dentro del campo (Levinas, 20I6), sin embargo, con una comunicación razonada se puede alcanzar una condición de enseñanza con la otra identidad sin tener límite alguno.

Así, "el babitus en última instancia se convierte en la forma en la cual una persona se adapta a ciertos esquemas y adecúa su comportamiento para cumplir con ellos por ser considerados como lo correcto" (Arévalo, 20I9, p. 66), es decir, es la disposición que hace que un ser humano se conduzca de una u otra forma. En la identidad (babitus) de los comerciantes, la comunicación interpersonal siempre está presente, porque cuando se hablan incorporan las disposiciones que han adquirido a través de la experiencia de cada uno de ellos, en diversos lugares y diferentes tiempos (Bourdieu, I988), de tal manera que con base a esas experiencias y a las preferencias de las personas existen lo bueno, lo útil y lo placentero, y en sentido contrario con base en las aversiones existen lo malo, lo nocivo y lo desagradable (Aristóteles, 2016).

Desde otra perspectiva, al integrarse las redes, la confianza y las normas, aspectos medulares del capital social, se parte de un enfoque inductivo, de lo micro a lo macro en un desarrollo que tiene por objeto articular los marcos individuales y colectivos, por tanto, el capital social apunta a los recursos que establecen las relaciones sociales, el sociólogo Robert Putnam distingue cuatro tipos diferentes de capital social, siendo: el capital social formal y el informal; el capital social denso y el tenue; el capital social vuelto hacia dentro y el vuelto hacia fuera; y el capital social vinculante y el que tiende puentes (2003). El último es el más importante para Putnam, el capital social vinculante refuerza las identidades tanto específicas como excluyentes, fortalece la correspondencia e incita la solidaridad, por su parte, el capital social que tiende puentes integra personas heterogéneas creando 
semejanzas y conexiones más amplias, implanta reuniones para intercambiar información con redes externas.

Por otra parte, Francis Fukuyama considera que el capital social "es un conjunto de valores o normas informales compartidas entre los miembros de un grupo, que permiten la cooperación entre ellos" (2000, p. 15). Por tanto, el capital social es un motor del desarrollo siempre y cuando se fundamente en la confianza social para que genere más confianza social y las sociedades puedan desarrollarse. En este último atributo, la confianza tiene que ser estratégica y moral (López C., 2020); la primera perspectiva es confiar a futuro en las expectaciones que se han establecido con antelación como patrón de comportamiento; la segunda perspectiva se refiere a poseer una actitud de beneplácito establecida en la educación sobre principios adquiridos.

En otro orden de ideas para Nan Lin, el capital social es información, influencia, credenciales sociales y refuerzo, el que posea estas particularidades, controla y funciona ese capital; "es visto como un activo social en virtud de las conexiones de los actores y el acceso a los recursos en la red o grupo del que son miembros" (pp. 19 y 20). En una estructura macrosocial que comprende posiciones con base a algunos recursos normativamente aceptados como la riqueza, el estatus y el poder, la estructura piramidal es en sí, una ordenación en un sentido de accesibilidad y control de los recursos, ejemplificando con una estructura modelo de un mercado móvil, cuanto más alta es la posición, menos ocupantes existirán. Independientemente del reconocimiento individual y las relaciones que se crean en el capital social, se pueden observar dos formas de capital social, uno individual o relacional donde los comerciantes que específicamente son integrantes de la red pueden emplear los recursos con base a las relaciones personales, y otro colectivo o sistémico, donde se crean recursos sociales como la confianza, cooperación, valores, principios morales, siendo una red amplia donde sin importar el nivel de participación y cooperación de los comerciantes (Hartmut, 2008), todos obtienen un beneficio.

\section{METOdología}

Habiendo analizado las investigaciones científicas sobre identidad (babitus) y el capital social; la intersubjetividad de los comerciantes; y su identidad (babitus) y capital social en los mercados móviles; y para saber cómo la identidad (babitus) y la intersubjetividad de ellos contribuye a la edificación del capital social en sus resoluciones, se establece la siguiente hipótesis: los comerciantes femeninos y masculinos tienen la misma participación en las asambleas delbazar. Lo anterior se considera partiendo del género de los comerciantes de los mercados 
móviles como parte de su identidad (babitus) e intersubjetividad, y del nivel de participación que tienen en las asambleas que realizan para decidir sobre asuntos que les concierne por ser miembros de una A.C., como parte del capital social. Para el caso se empleó la metodología mixta que ayuda a tener una configuración amplia y profunda del fenómeno en estudio, condensando las aproximaciones cuantitativas y cualitativas para lograr un conocimiento sistémico, completo y holístico (Lieber y Weisner, 20IO). Se encuestaron durante los meses de septiembre de 2020 a febrero de 202 I a 152 comerciantes del Bazar del Oro (BO) de una población aproximada entre 240 y 250 comerciantes; asimismo, en el Bazarte Obregón (BteO) la muestra que se encuestó fue de II5 comerciantes de una población entre I50 y I6o comerciantes; ambas muestras se obtuvieron empleando el programa Decision Analyst STATS.

La selección de los encuestados fue a conveniencia del investigador, es decir, la muestra es por conveniencia y la probabilidad de haber sido seleccionado para la muestra fue a decisión del investigador con fundamento en que la población es homogénea y los entrevistados son semejantes a la población definida total. Este tipo de muestreo permite encuestar a un gran número de entrevistados en un tiempo breve; asimismo, el tamaño del margen de error fue detectado en relación con las predicciones de la investigación, buscando asegurar una selección sin desvíos de las unidades muéstrales (Otzen y Manterola, 20I7), en otras palabras, la unidad fueron los comerciantes que integran los bazares quienes interactúan de diversas maneras, representando apropiadamente la muestra de acuerdo con la población de cada uno de los bazares, por tanto, el muestreo fue no probabilístico por conveniencia. Al reunir las respuestas de la encuesta se empleó el Paquete Estadístico para las Ciencias Sociales (SPSS), donde se aplicó la herramienta estadística $t$ de Student.

En la misma temporalidad se realizaron entrevistas en profundidad, cinco a comerciantes que pertenecen al BO y de la misma manera cinco a comerciantes que pertenecen al BteO. En este método, se siguieron lineamientos que orientaron al investigador, no se impusieron técnicas o procedimientos, sino se buscó la definición del mundo de los entrevistados por medio de la conducta, por medio de lo que platicaron y por medio de lo que hacen, apartando las creencias, perspectivas y predisposiciones del investigador (Taylor y Bogdan, 1994), en este proceso se interpreta, es decir, se ven las cosas que ven otras personas desde un enfoque inductivo.

\section{RESULTADOS}

De acuerdo con la información recabada por los comerciantes encuestados la hipótesis nula se acepta en el вО al contar con un nivel de significancia de la prueba estadística $t$ de 
Student de .88I, es decir, tanto comerciantes femeninos como masculinos, participan por igual en las asambleas de su A.C. En cambio, en el BteO se rechaza al contar con un nivel de significancia de .oor, esto significa que tanto comerciantes femeninos como masculinos no participan por igual en las asambleas de su A.C. La Tabla I muestra la significancia $.88 \mathrm{I}>.05$ en el вО aceptando la participación; por el contrario, la significancia .00I<.05 en el BteO rechaza la participación.

TABLA 1

\begin{tabular}{|l|c|l|l|c|}
\hline MERCADO MÓVIL & $\begin{array}{c}\text { COMERCIANTES } \\
\text { ENCUESTADOS }\end{array}$ & $\begin{array}{c}\text { COMERCIANTES } \\
\text { ENCUESTADOS POR GÉNERO }\end{array}$ & $\begin{array}{c}\text { MEDIA POR } \\
\text { GÉNERO }\end{array}$ & $\begin{array}{c}\text { SIGNIFICANCIA } \\
\text { PRUEBA DE LEVENE }\end{array}$ \\
\hline Bazar del Oro & 152 & $\begin{array}{l}\text { Femeninos: } 81 \\
\text { Masculinos: } 71\end{array}$ & $\begin{array}{l}\text { Femeninos: } 1.60 \\
\text { Masculinos: } 1.59\end{array}$ & .881 \\
\hline Bazarte Obregón & 115 & $\begin{array}{l}\text { Femeninos: } 74 \\
\text { Masculinos: } 41\end{array}$ & $\begin{array}{l}\text { Femeninos: } 2.14 \\
\text { Masculinos: } 2.32\end{array}$ & .001 \\
\hline
\end{tabular}

FUENTE: IBM SPSS CON DATOS PROPIOS, 2020 Y 2021.

Conforme con la información recabada por los comerciantes entrevistados en el BО se presentan algunas contradicciones. Un informante manifiesta que "existe comunicación y respeto, pero no se ve una participación en las asambleas, tratamos de mantener la unión de los pocos o muchos comerciantes que somos, pero muchos no asisten" (CMiBBO, 2020); en cambio, otro informante expresa que "el líder ha ido en decadencia al retirarle privilegios la alcaldía, eso ha ocasionado que el líder ya no vea por los comerciantes del bazar y anteponga sus intereses, con esto, todos los comerciantes cordialmente nos saludamos y respetamos, porque tampoco nadie quiere problemas, pero ya nadie va a las asambleas" $\left(\mathrm{CM}_{2} \mathrm{HBO}\right.$, 2020); otro informante establece "no hay un liderazgo como tal, la realidad es que en algún momento sí lo hubo, pero se prestó a mucha corrupción, pedían apoyos económicos que nunca se transparentaron cómo se usaron, por eso muchos compañeros no asisten a las asambleas" ( $\left.\mathrm{CM}_{3} \mathrm{JBO}, 2020\right)$; en ese mismo sentido, otro informante coincide con el anterior diciendo "pues la organización es muy pésima, ya que no se tiene una buena organización porque están muy divididos, todos quieren tener el poder y no jalan parejo, no van hacia un mismo fin, todo el mundo quiere lucrar y entonces no ven el bienestar del bazar, entonces esa es la realidad por la que el bazar no tiene un buen liderazgo, no hay una buena organización para poder llevar a cabo bien las asambleas" ( $\left.\mathrm{CM}_{4} \mathrm{BBO}, 2 \mathrm{O} 20\right)$; por su parte, 
un informante concuerda que "la organización está muy mal, eso sí, está mal de un par de años hacia la fecha, el o los líderes, porque ya no sabe uno cuántos son, no se organizan para llevar bien el bazar porque se la pasan peleando por desacuerdos, y cuando quieren hacer las asambleas nadie asiste, porque nadie quiere problemas" ( $\mathrm{CM}_{5} \mathrm{CBO}, 2 \mathrm{O}$ I). La Figura I muestra la nube de ideas donde aparecen las palabras clave con mayor frecuencia.

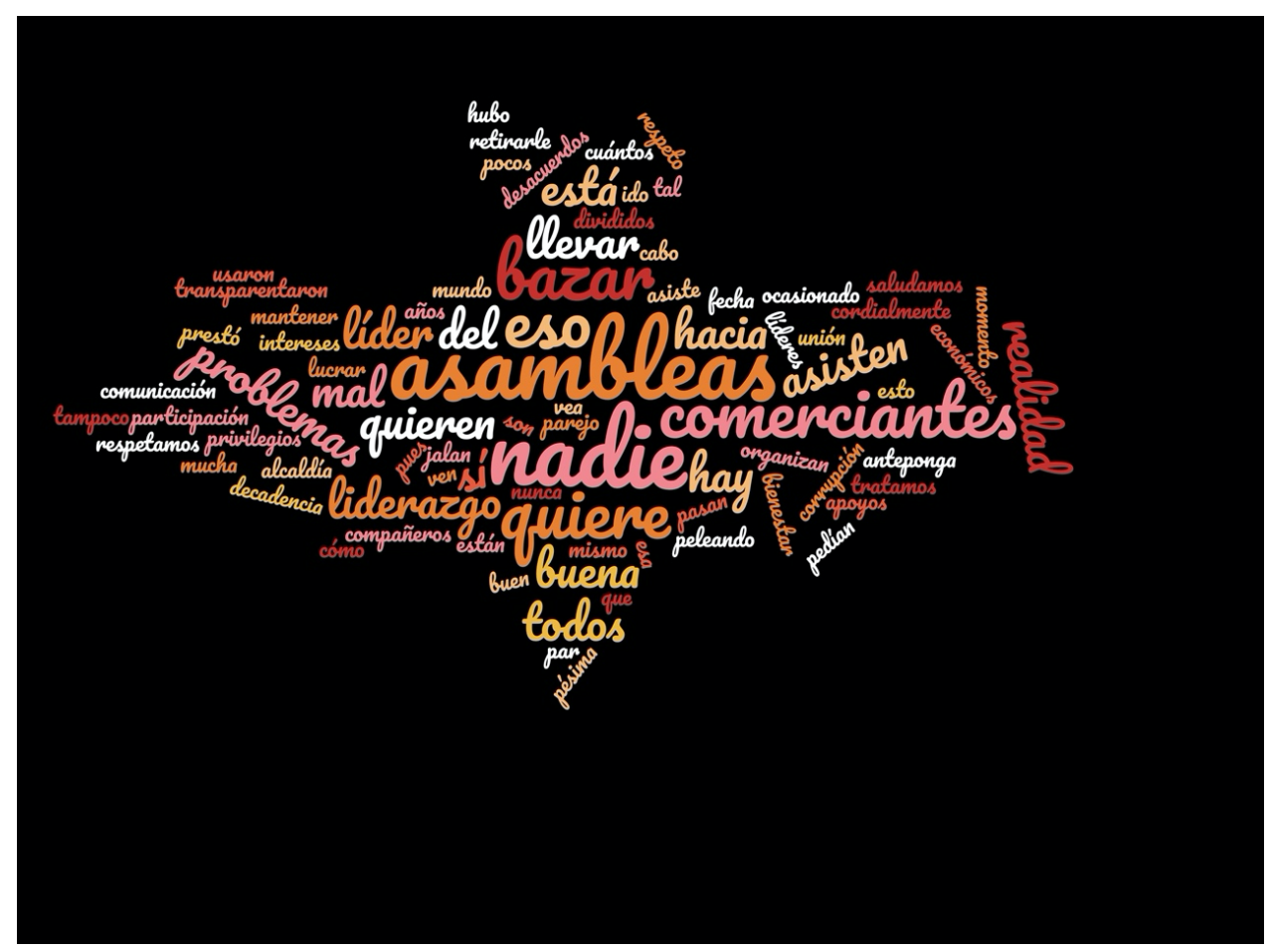

FIGURA 1. FUENTE: ELABORACIÓN PROPIA, 2021.

Acorde con la información recabada por los comerciantes entrevistados en el BteO se presenta una situación distinta, el informante establece que "está bien organizado todo, nuestra líder lleva su batuta como tal, y pues no es que no tengo nada de qué quejarme, asisto a las asambleas, porque sé qué mi voz va a ser escuchada” (CMiABteO, 202I); otro informante coincide diciendo que "la organización es muy buena, cuando asisto a la asamblea la líder es muy clara y apoyo lo que es bueno, ella es una persona buena y siempre nos apoya” (CM2PBteO, 202I); la opinión también similar de un informante es que "la líder se encarga de todo, a mí se me atora algo y ella me lo soluciona, nunca nos falta al respeto y cuando hay asamblea, asistimos todos y siempre pone las cosas a votación” ( $\mathrm{CM}_{3} \mathrm{CBteO}, 2 \mathrm{O}$ I); asimis- 
mo, un informante expresa que la organización "es muy profesional, cuando se participa en las asambleas se sabe sobre los problemas o asuntos que se van a tratar, se otorga el espacio para expresarnos y comunicarnos" ( $\left.\mathrm{CM}_{4} \mathrm{VBteO}, 2 \mathrm{O} 2 \mathrm{I}\right)$, así también, un informante manifiesta "hay coordinación y cuando se hacen asambleas la seriedad se vislumbra, podemos manifestarnos, comunicarnos y votar a favor y en contra, el resultado es palpable por los años que tiene el bazar" (CM5 LBteO, 202I). La Figura 2 muestra la nube de ideas con las palabras clave más frecuentes.

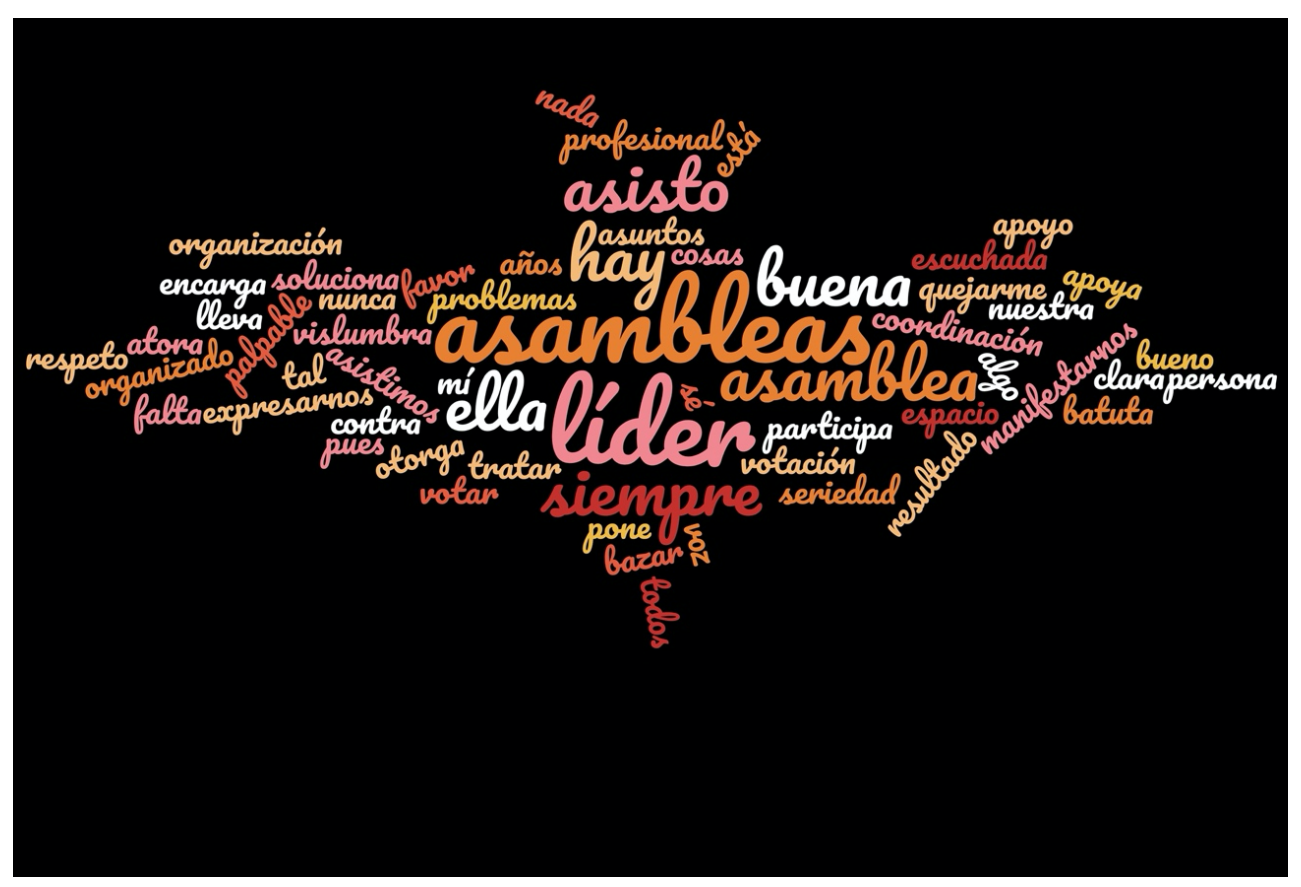

FIGURA 2. FUENTE: ELABORACIÓN PROPIA, 2021.

Con fundamento en las entrevistas en profundidad que se realizaron, se empleó una taxonomía para identificar a los informantes de ambos bazares con el objetivo de respetar su decisión de anonimato y no comprometer sus identidades y respuestas. La Tabla 2 establece la clasificación. 
TABLA 2. TAXONOMÍA DE INFORMANTES DEL BAZAR DEL ORO Y EL BAZARTE OBREGÓN

\begin{tabular}{|l|l|}
\hline Informante uno & $\mathrm{CM} 1 \mathrm{BBO}$ \\
\hline Informante dos & $\mathrm{CM} 2 \mathrm{HBO}$ \\
\hline Informante tres & $\mathrm{CM} 3 \mathrm{JBO}$ \\
\hline Informante cuatro & $\mathrm{CM} 4 \mathrm{BBO}$ \\
\hline Informante cinco & $\mathrm{CM} 5 \mathrm{CBO}$ \\
\hline Informante seis & $\mathrm{CM} 1 \mathrm{ABte} 0$ \\
\hline Informante siete & $\mathrm{CM} 2 \mathrm{PBte} 0$ \\
\hline Informante ocho & $\mathrm{CM} 3 \mathrm{CBte} 0$ \\
\hline Informante nueve & $\mathrm{CM} 4 \mathrm{VBte} 0$ \\
\hline Informante diez & $\mathrm{CM} 5 \mathrm{LBte} 0$ \\
\hline
\end{tabular}

FUENTE: ELABORACIÓN PROPIA, 2021.

\section{ANÁLISIS Y DISCUSIÓN}

Con base en los resultados encontrados de los comerciantes encuestados y entrevistados, se puede apreciar que existe una correlación inversamente proporcional entre las investigaciones cuantitativas y cualitativas respectivamente. Por mejor decir, mientras en el BO la investigación cuantitativa acepta la hipótesis nula de que los comerciantes de ambos géneros tienen la misma participación en las asambleas del bazar, las respuestas en la investigación cualitativa de las entrevistas en profundidad muestran que no existe una participación homogénea en las asambleas que se realizan. Por su parte, en el BteO la investigación cuantitativa rechaza la hipótesis nula de la misma participación de los comerciantes de ambos géneros en las asambleas del bazar, sin embargo, las respuestas en la investigación cualitativa de las entrevistas en profundidad muestran todo lo contrario, siendo que existe un empeño en participar activa y homogéneamente en las asambleas que se realizan.

En los mercados móviles las redes sociales, la confianza y normas establecidas por los líderes y comerciantes articulan los marcos individuales y colectivos, es decir, se parte de lo individual a lo general para tener una interacción con las personas, entre ellos y con la estructura social establecida (Vásquez-González, 20I8), en el BO estos marcos están consolidados entre el líder y los comerciantes, en cambio, en el BteO no se presenta esta situación, la cual influye a la colectividad. Sin embargo, en ambos bazares se establece un espacio social donde a partir de la interacción simbólica y la comunicación se genera un marco de referencia com- 
partido entre los comerciantes (Gómez y Herranz, 20I6) definiéndolo por los principios de significado como la reacción de acuerdo al sentido que se les da a las personas o cosas; el lenguaje como interacción social entre personas; el pensamiento como modificación de la interpretación simbólica; y el self como la capacidad de mirarnos como objeto y reflexionar sobre nosotros desde la perspectiva de otros (Blumer, I969; Griffin, 2009; Mead, 1962; Ritzer, 2007; West y Turner, 2005). En el BO estos principios configuran un accionar de falta de participación en las asambleas por parte de los comerciantes posiblemente por considerar que, al contar con una estructura social firmemente constituida, nadie asiste a pesar de los problemas existentes y la ausencia de liderazgo. En cambio, en el $\mathrm{BteO}$ los mismos principios configuran un accionar para participar en las asambleas por parte de los comerciantes, quienes apoyan a su líder y asisten, probablemente por la falta de esa estructura social que influye la organización, pero con la buena voluntad y coordinación para solucionar los problemas colectivos. Tanto la estructura social como los cuatro principios de la interacción contribuyen en la formación de comunidades. A partir de la comunicación, como reflejo de los mismos comerciantes, se van construyendo actitudes en la comunidad que a su vez generan una propia y exclusiva entidad fundamentada de lo que simboliza el во y $\mathrm{BteO}$ para sus integrantes.

\section{CONCLUSIONES}

Las experiencias compartidas en un espacio y una temporalidad por los comerciantes integrantes de cada uno de los bazares examinados conectan dimensiones de su propia estructura social, emanados de las interacciones que se producen entre ellos por la intersubjetividad que se genera y que se expresa por medio de la comunicación interpersonal para comprender diversas situaciones tanto reveladoras como determinantes; cada una de las conductas que se presentan están intervenidas por fuerzas de valores, normas, representaciones, costumbres y reglas sociales (Gadea, 20I8), es decir, por las disposiciones que se adquieren dentro de cada bazar y que se manifiesta por la identidad (babitus) de cada uno de sus comerciantes, distinguiendo la forma de observar el ámbito, así como la manera de proceder dentro de ese ámbito. La interacción social que se produce proporciona a los comerciantes de cada uno de los bazares los significados y símbolos peculiares que les habilitan la forma de conducirse y relacionarse, es por eso por lo que las prácticas sociales de uno y otro bazar difieren al momento de participar en sus respectivas asambleas.

Como consecuencia de la interacción social estructurada, se presenta entre los comerciantes de cada uno de los bazares una interacción simbólica que organiza un procedimiento de entendimientos sociales conocidos que se definen por el significado, el lenguaje, el 
pensamiento y el self; estos principios establecen interacciones y símbolos que dan forma a una realidad donde el actuar de las personas significan algo, en otros términos, los comerciantes analizan y delimitan el actuar de otros comerciantes otorgándoles argumentos con fundamento en los significados que les conceden a esas acciones (Blumer, 1969), dándole un entendimiento a esa realidad social incluyendo sus disyuntivas, contrariedades y reciprocidades, así, el interaccionismo simbólico es inseparable de la vida cotidiana dentro de los bazares de donde nacen los diferentes significados de la interacción social.

En los resultados de la investigación cuantitativa y cualitativa los comerciantes de cada bazar difieren significativamente porque, por una parte, están sujetos a una estructura social con prácticas, sentidos y valores organizados, vividos y representados (Gadea, 20 I8); en la investigación cuantitativa en el вO la estructura social impone que comerciantes femeninos y masculinos participen por igual en las asambleas; en ese mismo sentido, en el BteO la estructura social objeta que comerciantes femeninos y masculinos participen por igual en las asambleas, es decir, existe participación por parte de los comerciantes, pero puede existir una mayor participación de comerciantes femeninos o de comerciantes masculinos. Por la parte de la investigación cualitativa, cada comerciante se presenta como un significante que se describe en un orden simbólico de la interacción social; los símbolos que se presentan en diversas situaciones estructuradas, vividas e interpretadas, conforman el mundo de la vida de los comerciantes (Arévalo G. , 20I9); para los comerciantes del BO ese mundo de la vida les simboliza no participar en sus asambleas por los problemas de organización que se presentan por ausencia de liderazgo, pero para los comerciantes del BteO ese mundo de la vida les simboliza participar en sus asambleas por la buena gestión que realiza su líder. Esto configura la conducta encubierta (Mead, 1982) de los comerciantes que observan sus respectivos bazares por medio de símbolos y significados interiorizados, fortaleciendo y debilitando el capital social respectivo.

\section{REFERENCIAS}

Arévalo, G. (2019). Construcción del objeto político para la participación: una mirada desde el interaccionismo simbólico y la fenomenología social de Alfred Schütz en diálogo con la perspectiva arendtiana. Analecta Política, 9(17), 250-272. https://doi.org/I0.18566/apolit.v9ni7.a04\%20

Arévalo, R. (2019). Comunicación integral para las organizaciones. Liderazgo y creación de valor. Comunicación Social. Ediciones y Publicaciones.

Aristóteles. (2016). Ética nicomaquea. (24. ㄹ ed.). Porrúa.

Blumer, H. (1969). La posición metodológica del interaccionismo simbólico. Prentice Hall.

Bourdieu, P. (1988). Cosas dichas. Gedisa. 
Bourdieu, P. (1990). Cuestiones de sociología. Grijalbo.

De Zan, J. (20I7). El reconocimiento como presupuesto del lenguaje, de la intersubjetividad y del sujeto moral. En Miguel Giusti (Ed.), El paradigma del reconocimiento en la ética contemporánea. Un debate en curso. Fondo Editorial.

DeVito, J. (2013). The interpersonal communication book. (13. - ed.). Pearson Education.

Dussel, E. (200I). Hacia una filosofía política crítica. Desclée de Brouwer.

Esquivel, M. (2019). La incidencia de las políticas culturales en la imagen urbana de Tepoztlán, Morelos [Tesis de maestría]. Universidad Autónoma del Estado de Morelos.

Feregrino, M., y Cadena, Y. (20I9). Trayectorias de trabajo informal, género y espacio público en la Ciudad de México. Revista Latinoamericana de Antropología del Trabajo, (5), I-27. http://www. ceil-conicet.gov.ar/ojs/index.php/lat/article/viewFile/551/386

Foucault, M. (2008). El orden del discurso. (I. ${ }^{\mathrm{a}}$ reimp.). Tusquets.

Fukuyama, F. (2000). The great disruption: Human nature and the reconstitution of social order. Simon $\&$ Schuster.

Gadea, C. (20I8). El interaccionismo simbólico y sus vínculos con los estudios sobre cultura y poder en la contemporaneidad. Sociología,33(95),39-64. http://www.sociologicamexico.azc.uam.mx/ index.php/Sociologica/article/view/I392/I288

García, M. (2015). Comunicación y relaciones interpersonales. Tendencias Pedagógicas, 2, I-I7. https://revistas.uam.es/tendenciaspedagogicas/article/view/tpig96.2.ARTooI

Garrido, L. (20II). Habermas y la teoría de la acción comunicativa. Razón y Palabra, I6(75), I-I9. http://www.razonypalabra.org.mx/N/N75/ultimas/38_Garrido_M75.pdf

Garza, F. (2019). Identificando clústers culturales en el centro de Monterrey. Revista Contexto, I3, 4I-56. http://contexto.uanl.mx/index.php/contexto/article/view/I60/I57

Garza, M. (2009). La importancia de la interacción y de la comunicación interpersonal e intersubjetiva en la formación de comunicólogos. En Fátima Fernández Christlieb y Marta Rizo García (Eds.), Nosotros y los otros: La comunicación bumana como fundamento de la vida social. Editoras Los Miércoles.

Gobierno de la Ciudad de México. (2019). Gaceta oficial de la Ciudad de México. https://data.consejeria.cdmx.gob.mx/portal_old/uploads/gacetas/45ofbgf5402b3049c9cb6e2453b4Ibc7.pdf?fbclid=IwARıgZNcONplVh6Ns7j_n6JNAorCAgEYPgJNN8TbOijmGJMqGOtLZFwCNga8

Gómez, B., y Herranz, A. (20I6). ¿Hablas o te comunicas? Un método proactivo para mejorar la comunicación interpersonal. (2. ${ }^{\mathrm{a}}$ ed.). Marge Books.

González, F. (20I9). Siete narrativas en torno a la metrópoli de Guadalajara. Revista Estudios sobre las Culturas Contemporáneas, 25, 9-37. https://www.culturascontemporaneas.com/culturascontemporaneas/contenidos/o2 Siete_narrativas.pdf

Griffin, E. (2009). Una primera mirada a la teoría de la comunicación. (7.ㄹ ed.). McGraw-Hill.

Habermas, J. (2014). Teoría de la acción comunicativa. (I. ㄹ reimp.). Trotta.

Hartmut, E. (2008). The two meanings of social capital. En Dario Castiglione, Jan W. Van Deth y Guglielmo Wolleb (Eds.), The Handbook of Social Capital. Oxford University Press. 
Hernández-Jorge, C., y De La Rosa, C. (2017). Habilidades comunicativas en estudiantes de carreras de apoyo frente a estudiantes de otras carreras. Apuntes de Psicologia, 35 (2), 93-IO4. https://idus. us.es/bitstream/handle/II44I/85227/663-I493-I-SM.pd??sequence $=$ I\&isAllowed =y

Jackson, E. (2019). Valuing the bowling alley: Contestations over the preservation of spaces of everyday urban multiculture in London. The Sociological Review, 67(I), 79-94. https://doi. org/IO.II77/0038026118772784

Levinas, E. (2016). Totalidad e infinito. Ediciones Sígueme.

Lieber, E., y Weisner, T. (20IO). Meeting the practical challenges of mixed methods research. En Abbas Tashakkori y Charles Teddlie (Eds.), Handbook of Mixed Methods Researcb. SAGE.

Lin, N. (200I). Social capital: A theory a social structure and action. Cambridge University Press.

López, C. (2020). El capital social en América Latina: los desafíos de la comunicación social en el siglo XXI. En Wilson Hernández (Ed.), Comunicación pública en Iberoamérica I. Fundación Interamericana de la Comunicación.

López, M. (20I8). La configuración del trabajo en el siglo XXI: el mercado Eloy Salmón y los movimientos moleculares del capital. Íconos. Revista de Ciencias Sociales, 22(3), 87-104. http://dx.doi. org/I0.17I4I/iconos.62.2018.3244

Mead, G. (1962). Espíritu, persona y sociedad. Ediciones Paidós.

Mead, G. (1982). Mente, uno mismo y sociedad. Universidad de Chicago.

Otzen, T., y Manterola, C. (2017). Técnicas de muestreo sobre una población a estudio. International Fournal of Morphology, 35(I), 227-232. https://scielo.conicyt.cl/pdf/ijmorphol/v35nI/art37.pdf

Putnam, R. (2003). El declive del capital social. Un estudio internacional sobre las sociedades y el sentido comunitario. Galaxia Gutenberg-Círculo de Lectores.

Ritzer, G. (2007). Teoría de la sociología moderna. (7. ㄹ ed.). McGraw-Hill.

Sánchez, Y. (2019). Contribución de las prácticas culturales en el desarrollo de capital social comunitario [Tesis doctoral]. Universidad Nacional de Trujillo.

Schütz, A. (1972). Fenomenología del mundo social. Ediciones Paidós.

Solomon, D., y Theiss, J. (2013). Interpersonal Communication. Routledge.

Stewart, J., Zediker, K., y Witteborn, S. (2004). Together: communicating interpersonally. (6. $\stackrel{\mathrm{a}}{\mathrm{e}}$ ed.). Roxbury Publishing Company.

Taylor, S., y Bogdan, R. (1994). Introducción a los métodos cualitativos de investigación. (2.. reimpr.). Ediciones Paidós.

Uribe, R., Gutiérrez-Ríos, M., Varga, Z., y Vives, M. (2020). El diálogo en la construcción de la intersubjetividad crítica. Actualidades Pedagógicas, (75), I83-204. https://doi.org/10.19052/ap. voli.iss 75.9

Vásquez-González, Y. (2018). Midiendo el capital social: una aproximación desde la participación ciudadana en gobiernos locales. Revista Española de Ciencia Política, (48), I03-128. https://doi. org/I0.21308/recp.48.04

West, R., y Turner, L. (2005). Teoría de la comunicación: análisis y aplicación. McGraw-Hill.

Esta obra está bajo Licencia Creative Commons Atribución-NoComercial-SinDerivadas 4.0 Internacional. 